\title{
Lectura, escritura, matemáticas y habilidades digitales en la escuela. Hacia la necesidad de alfabetizaciones académicas continuas
}

Reading, writing, mathematics and digital skills at school. Towards the need of continuous academic literacies

\author{
Volumen 21, Número 1 \\ Enero - Abril \\ pp. $1-22$
}

\author{
Yolanda González de la Torre \\ José Jiménez Mora
}




\title{
Lectura, escritura, matemáticas y habilidades digitales en la escuela. Hacia la necesidad de alfabetizaciones académicas continuas
}

\author{
Reading, writing, mathematics and digital skills at school. Towards the need of continuous \\ academic literacies
}

\section{Yolanda González de la Torre ${ }^{1}$ José Jiménez Mora²}

\begin{abstract}
Resumen. La experiencia de las personas autoras como docentes en niveles que van desde la educación media hasta el posgrado permite notar deficiencias en el estudiantado vinculadas a ciertos saberes básicos. Es por eso que, en este ensayo, se discute acerca de la necesidad de que en cada nivel educativo se retomen tales saberes para facilitar la adquisición y construcción de conocimiento mediante procesos que, en la literatura, se pueden explicar desde el concepto alfabetización académica, de manera particular en cuatro aspectos: lectura, escritura, matemáticas y uso de las tecnologías de la información y la comunicación. El argumento principal es que al tratarse de saberes básicos que se esperaría tuviera consolidado el estudiantado en cada nivel, el hecho de que no los demuestre da lugar a la necesidad de definir, $y$, de ser necesario, ayudarlo a aprender tales saberes. Se concluye que, con ello, se podría mejorar su desempeño en tareas de adquisición y construcción de conocimiento según lo que le sea demandado en el nivel que se encuentre, así como a familiarizarse con requisitos de los campos disciplinares en que se forma, en el caso de las personas que están en licenciatura y posgrado
\end{abstract}

Palabras clave: lectura, escritura, matemáticas, alfabetización informacional.

Abstract. Authors' teaching background ranging high school to postgraduate education allow to notice students' lack of basic knowledge. Therefore, it is argued the need to recover that basic knowledge at each educational level to facilitate the acquisition and construction of knowledge, through processes that can be explained in the literature from the concept academic literacy, focused in four aspects: reading, writing, mathematics and use of information and communication technologies. The main argument holds that when dealing with basic knowledge that students would be expected to have consolidated at each level, the fact that they are not demonstrated gives rise to the need to define and, if necessary, help students learn such knowledge. It is concluded that this could improve their performance in tasks of knowledge acquisition and construction according to what is demanded of them at the level they are in, as well as becoming familiar with the requirements of the disciplinary fields in which they are trained, in the case of who are undergraduate and graduate.

Key words: reading, writing, mathematics, information literacy.

\footnotetext{
1 Profesora e Investigadora de la Universidad de Guadalajara, en el Departamento de Estudios en Educación, Guadalajara, México. Doctora en Educación por la Universidad de Guadalajara, Guadalajara, México. Dirección electrónica: ygtorre@hotmail.com Orcid https://orcid.org/0000-0003$\underline{0110-0030}$

2 Labora para la Secretaría de Educación Pública, Ciudad de México, Mexico. Maestro en Investigación en Ciencias de la Educación por la Universidad de Guadalajara, Guadalajara, México. Dirección electrónica: pepe dw@hotmail.com Orcid: https://orcid.org/0000-0003-4120-8724
}

Ensayo recibido: 27 de junio, 2020

Enviado a corrección: 18 de setiembre, 2020

Aprobado: 9 de noviembre, 2020

Los contenidos de este artículo están bajo una licencia Creative Commons 


\section{Introducción}

Actualmente, frente a las necesidades individuales y sociales que surgen en el desempeño de un oficio o profesión, las personas han tenido que hacer uso de los recursos con los que cuentan adaptando lo que ya conocen a nuevos escenarios o demandas y desarrollando otros para atender una tarea. Entre esos recursos se encuentra el capital lector o conjunto de habilidades para la lectura y las habilidades de cada persona para escribir de acuerdo con los distintos contextos en que lo requiere. Lo mismo sucede con el conocimiento matemático.

Los objetos de conocimiento abordados en este trabajo, además de la lectura y las matemáticas, incluyen la escritura y el uso de habilidades digitales. En principio, se propone, como premisa sobre estos objetos, la existencia de una brecha entre aquello que las personas aprenden en la escuela y lo que les es demandado en sus otros contextos de actuación. Al tratarse de un conjunto de saberes básicos para poder alcanzar un desempeño mínimo aceptado por la comunidad social a la que alguien pertenece, es posible reconocer que el proceso de adquirir tales saberes corresponde a una forma de alfabetización, mediante la cual las personas se familiarizan con ellos y con las tareas asociadas, alcanzando cierto grado de suficiencia en su ejercicio.

Este ensayo tiene como propósito principal aportar a la discusión y hacer notar cómo existen algunas evidencias de que varios de esos saberes se encuentran ausentes o poco consolidados en el estudiantado de educación preuniversitaria, lo que en México corresponde al nivel medio superior o bachillerato, y de educación universitaria, que abarca los niveles de licenciatura y posgrado. Se reflexiona sobre cuatro áreas de conocimiento consideradas fundamentales para el aprendizaje de asignaturas o disciplinas según el nivel: lectura, escritura, matemáticas y uso de las Tecnologías de la Información y la Comunicación (TIC). A esto último, se le denomina, en ocasiones, como "habilidades digitales", en el entendido de que lo aprendido sobre las TIC se traduce en desempeños que deberían resultar eficientes para las demandas de la actividad escolar.

El presente documento se compone de tres momentos (como se muestra en la Figura 1), en el primero de ellos se caracteriza la definición del concepto de alfabetización académica, posteriormente se discute sobre las dificultades y las demandas requeridas en los ambientes escolares de cuatro objetos de conocimiento: la lectura, la escritura, las matemáticas y las habilidades digitales, finalmente se exponen orientaciones generales como propuesta para propiciar la formación del estudiantado. 
Figura 1

Esquematización del documento

Inclusión de argumentos sobre demanda escolar-ayuda docente

\section{Alfabetización académica}

Caracterización del concepto

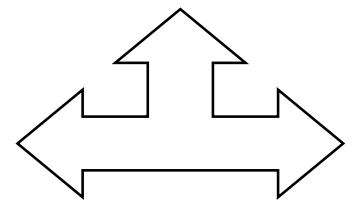

Objetos de conocimiento
Orientaciones generales para formación

\begin{tabular}{llll}
\hline Lectura & Escritura & Matemáticas & Habilidades digitales
\end{tabular}

Fuente: Elaboración propia, 2020

Sin pretender un análisis específico de esas cuatro áreas u objetos de conocimiento (ya que, por no tratarse de un reporte de investigación, no se cuenta con datos de campo) se acude a observaciones producto de nuestra experiencia en la enseñanza preuniversitaria y universitaria para ilustrar la cuestión que se aborda: la ausencia de saberes previos que se tiene que enfrentar cuando el estudiantado no es capaz de recordar ni aplicar, por ejemplo, procedimientos básicos de lectura o interpretación de datos numéricos.

\section{Alfabetización académica y su vinculación con la actividad escolar. Áreas de conocimiento consideradas}

Cuando se habla de alfabetización en general, el concepto connota la adquisición y puesta en práctica de conocimientos con algún sustrato teórico y otros saberes vinculados a procedimientos más de uso común que no necesariamente exigen poseer los fundamentos de tipo especializado. Así, por ejemplo, en el campo de lo que podría denominarse como "alfabetización digital", una persona puede reconocer conceptos como "correo electrónico", junto con otros asociados, como "contraseña", pero como usuario no le es requerido tener el dominio de las partes que constituyen la configuración interna de un protocolo informático.

De manera más precisa, en el caso de los conjuntos de conocimientos y saberes que se circunscriben a un escenario, como la escuela, en el que se formaliza su estudio, su aprendizaje puede ser denominado "alfabetización académica", concepto que como aclaran Álvarez-Álvarez y Boillos-Pereira (2015, p. 74), proviene de estudios ingleses, en el idioma español autores como Paula Carlino hace referencia a las actividades que las instituciones 
universitarias realizan para enseñar a leer y escribir al estudiantado según demandas específicas de las disciplinas en que se forman.

En ese sentido, la reflexión que aquí se expone no sostiene que el estudiantado y/o el profesorado no sepan leer, escribir, hacer matemáticas o acceder a recursos digitales, sino que durante el estudio de temáticas disciplinares de los programas escolares se van imponiendo necesidades específicas de conocimiento y desempeño para las cuales no basta con lo aprendido hasta cierto momento de la formación escolar. Ejemplos de estos saberes en los cuales se encuentran insuficiencias estudiantiles (como se mostrará más adelante) son lectura y matemáticas al interpretar datos, en matemáticas y escritura para respetar ciertas reglas sintácticas y en el manejo de recursos digitales al reconocer rutas de acceso a determinados sitios o documentos. Este tipo de saberes particulares son los que señalamos que se deben enseñar y aprender de manera específica para conseguir su dominio básico o "alfabetización".

\subsection{Exigencias de desempeño como indicadores de necesidad de saberes básicos en los niveles educativos}

Como se muestra de manera integrada en la Figura 2, la alfabetización académica se propicia a partir de determinadas prácticas de enseñanza que incluyen componentes como los cuatro objetos de conocimiento mencionados en este trabajo: lectura, escritura, matemáticas y uso de recursos digitales. Los ámbitos o niveles escolares en que tienen lugar tales prácticas comprenden un universo de exigencias y condiciones para los cuales la alfabetización académica configura determinados desempeños relacionados con el conocimiento, así, por ejemplo, para los niveles hasta el pregrado, la meta es que se adquieran las nociones básicas de un corpus de disciplinas incluidas en los programas de estudio; para el nivel de grado (licenciatura), la meta es la apropiación de conocimiento especializado y para el posgrado, se pretende que el estudiantado alcance el dominio de medios de producción y comunicación del conocimiento, como aquellos que son producto de la formación para la investigación. 
Figura 2

Alfabetización académica, evolución y fines de conocimiento

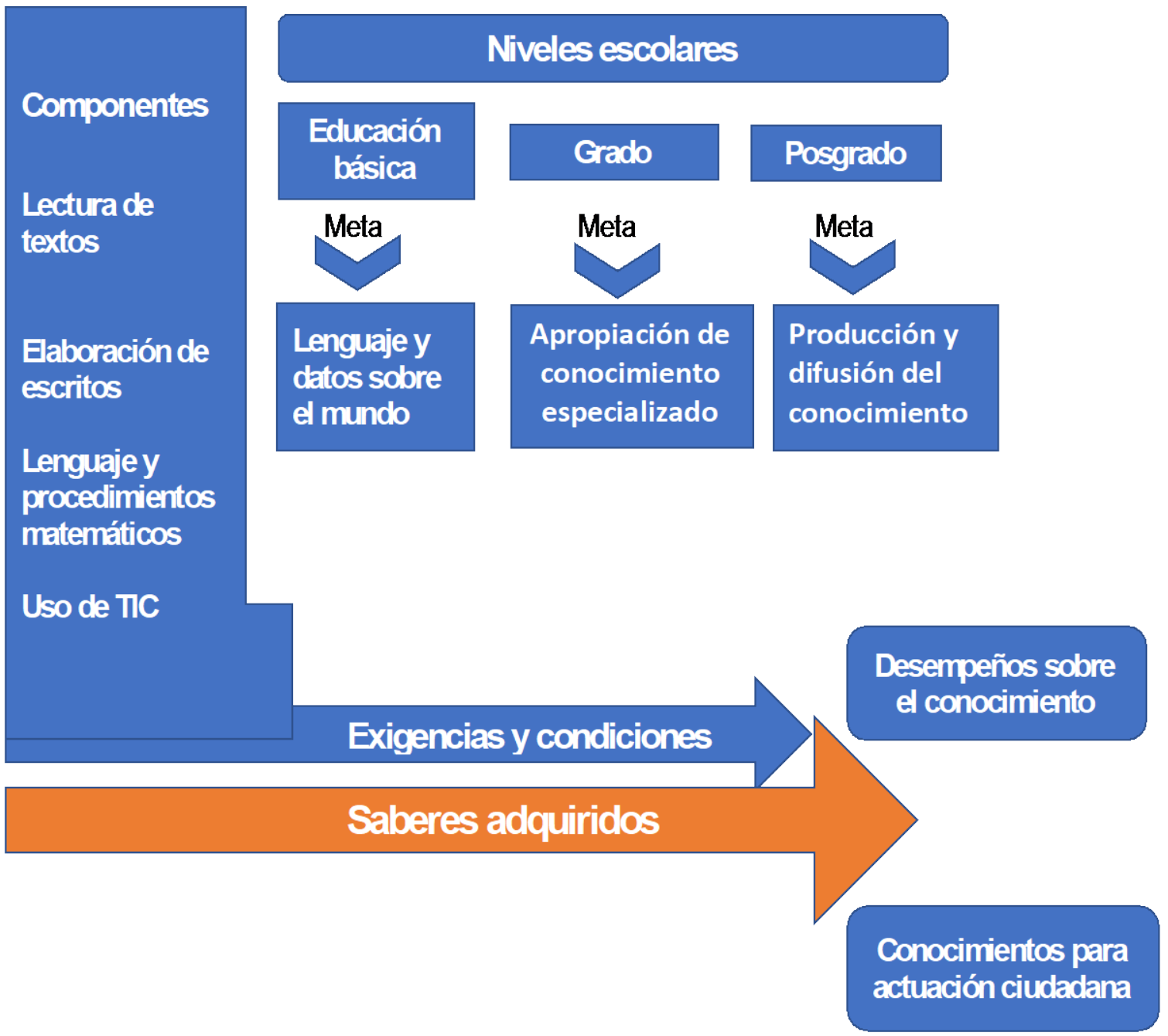

Fuente: Elaboración propia, 2020

Sin embargo, en cada nivel educativo estos componentes presentan una influencia gradual, puesto que los aprendizajes comprenden distinto nivel de complejidad y aquello que se pide al estudiantado también. En la Tabla 1 se presenta, de modo general, cómo los referentes se concretan en fines y producciones del estudiantado. 


\section{Tabla 1}

Progresión de la demanda en la alfabetización académica

\begin{tabular}{|l|l|}
\hline Lectura (de manera predominante qué, cómo y para qué se lee) \\
\hline Pregrado & $\begin{array}{l}\text { Texto expositivo y narrativo, énfasis en información básica para introducirse a } \\
\text { conocer un tema y documentar tareas escolares. }\end{array}$ \\
\hline Grado & $\begin{array}{l}\text { Texto expositivo y argumentativo, énfasis en comprensión de enunciados } \\
\text { conceptuales y técnicos para mostrar dominio profesional. }\end{array}$ \\
\hline Posgrado & $\begin{array}{l}\text { Texto argumentativo enfocado en diálogo crítico para crear ideas propias y trabajar } \\
\text { en su demostración. }\end{array}$ \\
\hline Escritura (qué se produce y cuáles exigencias tiene) \\
\hline Pregrado & $\begin{array}{l}\text { Trabajos de carácter monográfico que impliquen búsqueda documental, aunque no } \\
\text { necesariamente de fuentes especializadas. }\end{array}$ \\
\hline Grado & $\begin{array}{l}\text { Trabajos con mayor rigor en cuanto a la reflexión personal y a las fuentes referidas, } \\
\text { así como información diversificada (teórica, numérica, empírica). }\end{array}$ \\
\hline Posgrado & $\begin{array}{l}\text { Trabajos con alta exigencia en redacción, originalidad, uso de referentes, } \\
\text { elaboración de planteamientos conceptuales y análisis de datos. }\end{array}$ \\
\hline Matemáticas (tipo de conocimientos y habilidades requeridos) \\
\hline Pregrado & $\begin{array}{l}\text { Nociones e instrumental simbólico, procedimientos y leyes generales de álgebra, } \\
\text { geometría, estadística y análisis (cálculo diferencial). }\end{array}$ \\
\hline Grado & $\begin{array}{l}\text { Aplicaciones específicas según áreas profesionales, y aumento de niveles de } \\
\text { complejidad de contenidos matemáticos. }\end{array}$ \\
\hline Posgrado & $\begin{array}{l}\text { Procesamiento de datos numéricos de múltiples variables y alta destreza para su } \\
\text { interpretación en relación con fenómenos en estudio. }\end{array}$ \\
\hline Uso de tecnologías de información y comunicación (competencias digitales) \\
\hline Pregrado & $\begin{array}{l}\text { Manejo de procesador de texto, hoja de cálculo, software utilitario, acceso a sitios } \\
\text { web y habilidades básicas para transmitir y descargar información. }\end{array}$ \\
\hline Grado & $\begin{array}{l}\text { Reconocimiento de validez de fuentes, empleo de plataformas web y } \\
\text { combinaciones de aplicaciones, elaboración de bases de datos. }\end{array}$ \\
\hline Posgrado & $\begin{array}{l}\text { Dominio de software para análisis de datos cuantitativos y cualitativos, gestión de } \\
\text { recursos multimedia de socialización del conocimiento. }\end{array}$ \\
\hline
\end{tabular}

Fuente: Elaboración propia, 2020

Así, la alfabetización académica demanda una suerte de aprendizajes similares a los que tienen lugar en la alfabetización inicial (la que nos permite decir que sabemos "leer, escribir y hacer cuentas"), los cuales, progresivamente, se van incrementando en amplitud y profundidad. Ambas alfabetizaciones, desde la perspectiva de este ensayo, tendrían que ser objeto de la educación escolar; sin embargo, en el caso de la alfabetización académica, consideramos que los resultados en el desempeño del estudiantado evidencian que falta mucho por hacer ${ }^{3}$, de ahí la noción de que los esfuerzos de enseñanza se deben dirigir a "continuas" alfabetizaciones académicas.

\footnotetext{
${ }^{3}$ Los resultados del Programa Internacional para la Evaluación de los Estudiantes (PISA, por sus siglas en inglés, en su última aplicación, reflejan nuevamente que nuestro país está por debajo del promedio de las naciones pertenecientes a la Organización para la Cooperación y el Desarrollo Económico (OECD, Resultados de PISA 2018, Volumen I-III, PISA, OECD Publicaciones, Paris, 2019).
} 


\subsection{Argumentos para la discusión de la problemática de los "saberes faltantes" en lectura, escritura, matemáticas y habilidades digitales}

En principio, un punto que resulta conveniente traer a la discusión corresponde a la disposición del estudiantado hacia el conocimiento que se incluye en el currículum de la escuela, particularmente desde la perspectiva y fines estudiantiles. ¿Para qué es necesario saber algo? ¿Qué tanto conocimiento es necesario aprender? ¿Qué tipo de uso se da al conocimiento en la escuela y fuera de ella? Son preguntas asociadas con lo anterior, mientras que cierto tipo de respuestas a estas preguntas permiten notar un pragmatismo presente durante las situaciones de enseñanza y aprendizaje del conocimiento escolar.

Un ejemplo de esa postura pragmática se muestra en las tareas de comprensión lectora y de escritura, en casos en que el estudiantado no muestra deseo de apropiarse de conocimiento, sino de cumplir un requisito, ante la necesidad de aprobar más que de aprender. Lo anterior se manifiesta en prácticas como lecturas breves y sencillas para efectuar tareas consistentes, en "llenar" cierta información, como responder cuestionarios o transcribir apuntes dictados por el profesorado, según los hallazgos reportados por Fernández y Carlino (2010). En niveles educativos, como el universitario, también se da esta tendencia. El estudiantado no se muestra capaz de pasar de la manipulación de información a su apropiación y a la elaboración de nuevas ideas. Así, la escritura es considerada un ejercicio para obtener buenas notas, más que una herramienta de aprendizaje (Carlino, 2017).

En ese mismo sentido, se puede aseverar que existen vicios de lectura y escritura, como "cortar y pegar", los cuales corresponden a formas no deseables de incorporar información a los textos solicitados como parte de su actividad escolar, porque al hacer eso no interviene una exploración y reflexión sobre la información, que permita hacer una elaboración sobre ella además de yuxtaponerla, como se evidencian en los estudios de Argüello $(2009,2012)$, entre otros.

En contraparte, la comprensión lectora como habilidad cognitiva nos permite alcanzar el significado global y elaborar una representación mental de lo que estamos leyendo (Cuschnir, 2016). Siguiendo a la autora citada, la decodificación es una condición necesaria, pero no suficiente para la comprensión de textos. Cuando se trata de pasar a otro nivel de comprensión, el estudiantado de todos los niveles muestra deficiencias en lo que se refiere a acciones, como localizar ideas centrales de un texto o realizar inferencias diversas (Ugarriza, 2006; Calderón-lbáñez y Quijano-Peñuela, 2010; Triana, 2015; Peredo, 2016; Guevara, Cárdenas, Reyes y González, 2017). 
En cuanto a la composición escrita, se ha encontrado, en el estudiantado, distintas dificultades para elaborar las ideas; para construir argumentos o conclusiones de un escrito; terminan por hacer textos desestructurados y sin una consideración de las personas lectoras a los que están destinados (Uribe-Álvarez y Camargo-Martínez, 2011; Álvarez-Álvarez y Boillos-Pereira, 2015). Igualmente, esto puede explicarse por el hecho de que algún personal docente, en ocasiones, no sabe especificar lo que requiere de lectura y escritura para sus cursos, con lo cual la orientación que ofrecen es escasa y la retroalimentación, mínima.

Como consecuencia, según apunta Carlino (2017), quienes tienen a su cargo la enseñanza universitaria cuestionan en qué medida deben hacerse cargo de solventar la falta de desempeños adecuados de lectura y escritura del estudiantado, que se supone debía haber consolidado en niveles de educación previa. Sin embargo, Moreno (2019) subraya, como parte importante de la alfabetización académica, la pericia de cada docente en su mediación con el estudiantado para la comprensión y producción de textos.

Cuestión aparte es el asunto de que el profesorado, particularmente de pregrado, disponga del nivel de competencia que garantice su enseñanza al estudiantado en estas áreas (lectura, escritura, matemáticas o manejo de recursos, digitales). En casos como la escritura académica y el dominio de los recursos digitales, hay evidencia de que buena parte del profesorado también experimenta dificultades (Serrano, Duque y Madrid, 2012; Ibarra, Escalante y Darío, 2014; Castro, 2015; Elías, 2018 y García, 2019).

Otro tópico de discusión, a partir de algunos resultados mostrados por estudiantes, cuyo desempeño ha sido evaluado mediante pruebas estandarizadas, corresponde a los bajos niveles de habilidad matemática contemplada en esos instrumentos ${ }^{4}$. Se trata de una dificultad compartida por las y los actores de la enseñanza y del aprendizaje. Con respecto a esa insuficiencia, la postura de este trabajo, en principio, es que la relación entre comprensión lectora y aprendizaje matemático es fundamental, de tal modo que si no existe la primera no se da el segundo.

Según explican distintas investigaciones (García-Madruga y Fernández-Corte, 2008; Backhoff, Sánchez, Peón y Andrade, 2010; Sandoval, Frit, Maldonado y Rodríguez, 2010; Cuschnir, 2016), el aprendizaje matemático exige razonamiento a partir de enunciados o proposiciones, tarea en la que intervienen procesos como la conexión de significados que

4 En el país, otros instrumentos aparte de la prueba PISA que se han aplicado a la población estudiantil preuniversitaria son la Evaluación Nacional del Logro en Centros Escolares (prueba ENLACE), denominada posteriormente Plan Nacional para la Evaluación de los Aprendizajes (prueba PLANEA). 
después son almacenados en la memoria de cada estudiante y que sirven para la asimilación de información nueva. No obstante, la práctica cotidiana de la enseñanza provee múltiples evidencias en las que se distingue la ausencia de procesos como el anterior. Una de ellas aparece cuando el estudiantado no comprende lo que se le pide en determinadas instrucciones, aunque estas se refieran a acciones que han realizado previamente.

De lo anterior se puede inferir que el texto condensa una base de significados que si no son interpretados por el estudiantado no permiten desencadenar procesos de resolución, tales como la apreciación de datos y relaciones. Distéfano, Pochulu y Font (2015), en ese sentido, analizan dificultades cognitivas ante tareas que implican expresiones donde se utiliza notación y representaciones matemáticas particulares. Como en el aprendizaje de las matemáticas el estudiantado enfrenta la necesidad de leer y de escribir símbolos (muchos de los cuales fuera de esa disciplina no tienen sentido), los autores mencionados resaltan la necesidad de que desarrollen como habilidad específica la lectura y la escritura de expresiones simbólicas matemáticas.

El estudio citado anteriormente toma como referencia los aportes de trabajos como el de Godino, Font y Wilhelmi (2008) donde se desarrolla una teoría sobre los objetos matemáticos compuestos por una serie de componentes vinculados entre sí: situaciones-problema, elementos lingüísticos, conceptos, proposiciones y argumentos. De acuerdo con esta teoría, el conjunto de expresiones de la disciplina matemática que la persona docente utiliza en el aula, son el soporte para comunicar las particularidades de los objetos matemáticos y para comprenderlos y resolver las tareas de aprendizaje se requiere de procesos como la inferencia lectora y la argumentación escrita.

En relación con lo anterior se ha encontrado, sin embargo, que, a pesar de que el estudiantado lee oralmente una expresión simbólica, no siempre es capaz de reconocer, evocar o crear un ejemplo de los objetos matemáticos implicados, que sea congruente con su significado. En otras palabras, el estudiantado puede realizar procedimientos sobre símbolos no comprendidos (Jiménez, 2011). Ideas y hallazgos como los mencionados sugieren que para comprender los objetos variados que se enseñan en las distintas ramas de la matemática (Aritmética, Álgebra, Geometría y Trigonometría, Estadística y Probabilidad, entre otras), requieren de acciones diferenciadas que se señalan enseguida:

- El reconocimiento de los símbolos y su evocación

- La generación de imágenes mentales

- El dominio de formas sintácticas propias de cada rama 
- La posibilidad de compartir, junto con grupos de aprendices, los significados dados a las expresiones simbólicas que se leen.

Esto resulta aún más complicado si se tiene en cuenta que los mismos símbolos, en ramas distintas de la matemática, deben leerse o interpretarse de manera distinta, por ejemplo:

La expresión " $\mathrm{f}(\mathrm{x})$ ", en las asignaturas de Cálculo Diferencial o Cálculo Integral no se debe leer "f por x", como correspondería de acuerdo con los conocimientos previos del estudiantado sobre Álgebra, sino "f de x", y designa un concepto clave: una función, que puede definirse como la relación entre dos variables.

La expresión " $y=m x+b$ ", en la asignatura de Geometría Analítica se utiliza para modelar el concepto "ecuación de la recta en forma pendiente y ordenada al origen"; no obstante, en otra asignatura, como Cálculo Diferencial o Integral, hace referencia a otro concepto: "función lineal".

Así, para aprender matemáticas no basta que el estudiantado incluya en su discurso lo que dicen los símbolos, se requiere también que se familiarice con los conceptos a los cuales tales símbolos refieren, y con las formas en que se utilizan. Esto es, que adquiera poco a poco habilidades de lectura y escritura matemática.

Es por ello que en áreas como la resolución de problemas de matemáticas, un efecto de las ausencias en la comprensión lectora es la imposibilidad del estudiantado para un manejo consolidado de la información, lo cual demanda la intervención docente para aclarar los conceptos involucrados tanto en el enunciado como en las rutas de solución (Cuschnir, 2016). Asimismo, sin este tipo de comprensión no se puede acceder a los conocimientos básicos de matemáticas para actuar en un contexto cotidiano, es decir, alcanzar la alfabetización matemática o "mathematical literacy" (Machaba, 2018).

Por otra parte, ante la llegada de los dispositivos móviles y la Internet, un hecho que enfrentamos quienes nos dedicamos a la enseñanza es la presencia de dificultades para controlar la atención en el estudiantado. En esto incide, de alguna manera, un fenómeno que podría describirse como "fugacidad de la información", el cual describimos como el hecho de que el estudiantado retiene pocos datos en su memoria sobre temas del conocimiento escolar que les son proporcionados en una clase. Una de las influencias que advertimos en este fenómeno es la relación del estudiantado con los dispositivos que contienen información 
digital, pues ante la posibilidad de acceder a información para necesidades inmediatas, sobre todo en Internet, ya no se vuelve necesario para ellos ejercitar su capacidad de recuerdo y evocación, es decir, su memoria. En este sentido, como muestran en su revisión de investigaciones Cabra-Torres y Marciales-Vivas (2009), se puede apreciar cómo estos "nativos digitales" no desarrollan otras habilidades más allá de aquellas que tienen que ver con el mero acceso a la información.

En concordancia con lo anterior, aunque hay quienes suponen que las y los "nativos digitales" no necesitan instrucción alguna para interactuar con la tecnología y cuentan, de manera casi natural con ciertas habilidades digitales básicas, otras posturas han señalado cómo la incorporación de las Tecnologías de la Información y de la Comunicación (TIC) al ámbito académico se ha dado de manera mecánica y con una visión pragmática (Aparici, 2002; Grasso, Pagola y Zanotti, 2017), lo cual da lugar a la discusión en torno a las expectativas sobre las TIC como recurso de aprendizaje.

Respecto a ese punto, el objetivo de este trabajo no es sostener que, de manera necesaria, los procesos de lectura, escritura y comprensión matemática se ven afectados por el contacto del estudiantado con las TIC, sino establecer un marco de discusión sobre posibles interacciones entre esos procesos y los objetos de conocimiento que concebimos (lectura, escritura, matemáticas y habilidades digitales) en el contexto de la enseñanza escolar.

Como ejemplo de lo anterior, consideramos que leer es una actividad en la que se ven expuestos hábitos del estudiantado en torno al procesamiento de información de forma individual, pero donde también se pueden ver reflejados rasgos que son producto de su participación en grupos de usuarios que han desarrollado modos similares de emplear las TIC. En este sentido resulta que, no es lo mismo mostrarse hábil para el traslado de imágenes, descarga y empleo de aplicaciones, o en el uso de medios de audio y video disponibles en la red y en los dispositivos móviles, lo cual puede ser muy importante para participar en un grupo de amigos, sin embargo, distinguir conceptos, enunciados y párrafos que, en su conjunto, expresan descripciones, narraciones, explicaciones o argumentos, son acciones requeridas para el desempeño en la escuela.

Sobre la comprensión lectora se pone en cuestión, por ejemplo, si en los contextos digitalizados hay una transformación de las habilidades y estrategias de lectura respecto a las que se emplean al leer texto impreso, o si solo se trata de un cambio de soporte para leer. Mientras una posición sugiere que la lectura de textos electrónicos demanda prácticamente las mismas habilidades que la lectura de textos impresos (Albero, 2002; Rocha, 2004; Lugo 
de Usategui, 2005; Apolo, Bayés y Hermánn, 2016), entre ellas localizar determinada información, reconocer significados y poner atención en la forma de construcción del texto, otra sostiene que la lectura en formato digital demanda habilidades más allá de las necesarias para la lectura impresa, las cuales no se hallan presentes en total de las y los "nativos digitales" (Cabra-Torres y Marciales-Vivas, 2009; Coiro, 2011; Flores-Carrasco, Díaz-Mujica y LagosHerrera, 2017).

Esos estudios explican una situación particular a discutir: como en la lectura digital es posible tener a la vista otras fuentes de información (imágenes, sitios web, redes sociales), la demanda de atender dos o más tareas divide la atención y cuidado con que se desarrolla, ocasionando una especie de interferencia, además de que las personas lectoras de texto electrónico no siempre saben reconocer fuentes de información pertinentes. Una conclusión de lo anterior es que las generaciones familiarizadas con el uso de las tecnologías de la información y la comunicación, a pesar de mostrar pericia en cuanto a ciertos aspectos técnicos o prácticos, como por ejemplo interactuar con varias pantallas con información de un tema, no necesariamente alcanzan dominio de otros aspectos vinculados a la comprensión lectora, como los de orden cognitivo, entre los que se encontrarían reconocer y diferenciar significados, realizar inferencias o identificar argumentos.

Los aspectos problemáticos señalados anteriormente, en la experiencia de la autora y el autor de este trabajo, se extienden a lo largo de niveles educativos diversos, desde la educación media superior hasta el posgrado. Sin embargo, parece privar una postura generalizada que concede grandes posibilidades a las TIC y al empleo de las habilidades en su uso como recurso de aprendizaje. En el Modelo Educativo de la Secretaría de Educación Pública de México (SEP) (2017), se declara, por ejemplo, la finalidad de incorporar las TIC en la enseñanza para que el estudiantado tenga opción de emplear en el aprendizaje sus "habilidades digitales", y en el caso del profesorado, para que se pongan a punto con ello, empleando diversas fuentes de información y recursos existentes en Internet, así como aplicaciones y software diseñados para tareas educativas.

No obstante, ese tipo de posturas obvia el hecho de que las habilidades digitales del estudiantado no abarcan la gama de necesidades del aprendizaje escolar, mientras que por otra parte reconoce que no todo el profesorado está familiarizado con el uso de recursos como aplicaciones y plataformas existentes. De esta manera, se revela la necesidad de una “alfabetización digital” (Burin, Coccimigglio, González y Bulla, 2016), que no se reduce al mero acceso a la información, sino que comprende otros procesos como los mencionados 
anteriormente, en términos de comprensión lectora. Por otra parte, Godoy (2017) distingue en el uso de las TIC para el proceso de enseñanza aprendizaje las formas en que se usan, otorgando un lugar preponderante a su utilidad didáctica, desde el supuesto de que las tecnologías no son neutras y con solo implementarlas no producen un mejora en el aprendizaje del estudiantado o en la práctica del profesorado.

En términos de la matemática que se enseña en la escuela, por ejemplo, esta discusión también aborda la naturaleza y fines del conocimiento contenido en los programas de estudio. De modo particular, trabajos como el de Machaba (2018) enfatizan la diferencia entre los contenidos curriculares de matemáticas y la base de conocimientos y habilidades de las que un ciudadano debería disponer para comprender su entorno, lo cual incluye saberes elementales, pero también menos descontextualizados respecto de los que son enseñados en los programas escolares. Sea que se trate de la disciplina de la Matemática (Mathematics) o de sus elementos fundamentales de razonamiento, que se podría traducir como "alfabetización matemática" (Mathematics literacy), el papel de lo que una o un sujeto es capaz de comprender en lo que lee, de lo que es capaz de escribir o de aquello que puede conseguir de las TIC es preponderante.

\section{Conclusiones}

La escuela es la institución de la cual se espera la formación de las personas para desempeños generales y especializados en la sociedad, tanto en lo que concierne a una función técnica o profesional determinada como a su actuación ciudadana. Para ello, se han almacenado conocimientos diversos sobre el mundo, que son organizados para su enseñanza a manera de asignaturas desde la formación preuniversitaria hasta la que concluye con una licenciatura, y en el caso de posgrado se organiza en seminarios o talleres. En la escuela se da forma concreta a la enseñanza a través de prácticas en las cuales son incluidas, de manera específica, la lectura, la escritura, las matemáticas y las habilidades digitales según el nivel del sistema educativo y el contexto particular de aprendizaje. Estos cuatro objetos de conocimiento, como han sido denominados en esta reflexión, constituyen una base para construir nuevos conocimientos de acuerdo con las asignaturas o disciplinas que se pretende sean aprendidas por el estudiantado. En otras palabras, para aprender cualquier conocimiento disciplinar (biología, historia, pedagogía, por señalar algunos), en la época actual, se requiere 
leer, escribir, comprender datos y procesos numéricos, así como hacer uso eficiente de tecnologías de información y comunicación. ${ }^{5}$

No obstante, durante la labor cotidiana de la enseñanza en el contexto donde surge esta reflexión se recoge la queja generalizada de que entre el estudiantado faltan conocimientos considerados básicos de lectura, escritura, matemáticas y habilidades para el uso de las TIC. La intención de este trabajo no ha sido determinar a qué se deben estas deficiencias, sino a establecer su existencia y dar cuenta de la emergencia de dos tareas de carácter docente: 1) Definir los saberes que debe poseer el estudiantado en cuanto a lectura, escritura, matemáticas y uso de las TIC para iniciar el aprendizaje de nuevo conocimiento disciplinar. 2) Dirigir al estudiantado a la toma de conciencia de la necesidad de esos saberes básicos y apoyar con ayudas pedagógicas pertinentes a su apropiación. De esta manera, se podrían integrar necesidades concretas de conocimiento con las prácticas de personas para apropiarse de él y comunicarlo en algún campo de desempeño. Es por ello que en este trabajo, se habla de alfabetizaciones académicas continuas. Los trabajos de investigación que se realicen en este sentido podrían aportar evidencia empírica para examinar, en términos de lectura, escritura, matemáticas y habilidades digitales, cuáles demandas tiene ahora el estudiantado y en qué medida se posibilitan o se limitan mediante ciertas prácticas escolares.

Considerando los modos en que se concretan estas demandas y los resultados que producen, en particular en relación con el uso de las TIC, cuando el estudiantado "confiesa" realizar acciones que suponen el mínimo esfuerzo, como no leer libros o textos completos en sus dispositivos sino solo información puntual, la lectura se reduce a la búsqueda de información temática, la escritura a la reutilización de fragmentos de textos y el aprendizaje matemático a una búsqueda de videos explicativos. Esta situación, si bien es una condición necesaria para responder a las demandas de la actividad escolar, no es suficiente para la conformación de una base de saberes de los cuales el estudiantado pueda disponer posteriormente para realizar tareas de mayor nivel de complejidad, como las que suponen, por ejemplo, la elaboración de informes, tesis o proyectos de trabajo, entre otras.

De modo particular, en lo que se refiere a los procesos de producción escrita, tanto los que corresponden a lenguaje verbal o los referidos a expresiones simbólicas del conocimiento matemático parecen sostenerse sobre copia de fragmentos de información, obtenida generalmente desde alguna página o sitio de Internet, los cuales son añadidos a los textos que

\footnotetext{
${ }^{5}$ Las habilidades digitales, por ejemplo, forman parte de lo que se ha incluido en propuestas como el llamado Nuevo Modelo Educativo en México (SEP, 2017) para lo cual se establecieron determinaciones curriculares específicas.
} 
se intenta elaborar básicamente por su similitud con lo que es requerido para determinadas tareas escolares, aunque desafortunadamente en varias ocasiones sin siquiera ser leído y mucho menos comprendida esa información. Lo anterior pareciera revelar el hecho de que la inclusión de las tecnologías solo cambia los modos de realizar ciertas prácticas en el estudiantado en torno a pautas de aprendizaje, como las de sustituir apuntes explicativos personales por información de menor jerarquía al copiar grandes extensiones de un texto para darle contenido a sus trabajos escritos, propensión que se vuelve mayor cuando se requiere procesar textos y, con base en ellos, realizar elaboraciones escritas, como han mostrado entre otros González, Jiménez y Rosas (2016).

A efecto de evitar este pragmatismo implícito en los nuevos modos del estudiantado para aproximarse al conocimiento donde sus habilidades digitales juegan un papel esencial, resulta indispensable dotar de sentido al conocimiento de los programas escolares, principalmente, en términos de la comprensión del mundo por parte de las personas, y en la medida de lo posible, de sus propias experiencias. Asimismo, el conocimiento que representa la lectura, escritura y las matemáticas, así como aquel al que permite acceder, tienen que ser tratados como objetos especializados de aprendizaje en todos los niveles escolares. De este modo, es viable desterrar prácticas poco deseables del estudiantado con acceso a las TIC, tales como la superficialidad de sus búsquedas de información ante la inmediatez que ofrecen estas tecnologías.

Debe contemplarse también, respecto a las categorías lectura, escritura, matemáticas y habilidades digitales, en qué medida se habla de la posibilidad del estudiantado para llevar a cabo con destreza y de manera reflexiva, determinadas tareas en el contexto general de su formación, así como tomar en cuenta hallazgos de los estudios revisados en este trabajo, que sugieren cómo las TIC y las "habilidades" relacionadas con ellas hacen emerger usos automatizados de información sin un filtro reflexivo que asegure la comprensión sólida de las tareas de lectura, escritura y razonamiento matemático que se realizan durante el aprendizaje escolar.

En cuanto a la base de conocimientos que el estudiantado logra como resultado de la educación escolar, un caso representativo de la necesidad de estas "nuevas alfabetizaciones" se da ante la incursión al estudio de una carrera universitaria que prepara para el desempeño profesional, pues allí se les demanda una nueva orientación del conocimiento. A diferencia de lo que ocurre en la educación preuniversitaria, en el contexto donde se desempeñan la y el autor de este ensayo, donde predominan prácticas de reproducción de información para 
"cumplir con el programa", en la universidad cada estudiante enfrenta el reto de apropiarse de información de disciplinas encaminadas al dominio de ciertos conocimientos y, en ocasiones, a la demanda de producirlos, como es el caso de algunos posgrados orientados a la investigación. De este modo, se da una suerte de inicio de ciclos de alfabetización académica, vista como un proceso que consiste en favorecer el acceso de estudiantes a las culturas de lectura, escritura y razonamiento de las disciplinas.

Particularmente, en el caso de la educación posterior a la educación básica, el propósito de la alfabetización académica es entonces que el estudiantado aprendan los modos de leer, escribir y razonar de especialistas, y a la vez, aprendan a apropiarse del conocimiento producido por ellos en todas las asignaturas o materias a lo largo de la formación, así como de las formas de comprenderlo, producirlo y comunicarlo. Sin embargo, eso contrasta en ocasiones con los fines y resultados de la enseñanza en niveles escolares previos.

En todo caso, un sistema de prácticas escolares en el que se propicia alfabetización académica, incluso en niveles preuniversitarios, tendría que considerar y traducir en actividades concretas objetivos como los siguientes:

1) Dotar las actividades escolares de sentido educativo más allá del pragmatismo que implica aprobar asignaturas. De esta manera, se puede hacer notar a quienes participan en tareas del aula cómo la lectura, la escritura, el conocimiento matemático y las habilidades para el uso de las TIC obedecen a posibilidades de formación, como el ejercicio de la ciudadanía.

2) Implicar al profesorado en la mejora de su práctica como diseñadores de situaciones de aprendizaje. Esto se traduce en demandas específicas: emplear instrucciones precisas en las actividades que delegan al estudiantado; generar las mediaciones más adecuadas para la comprensión de los contenidos; insertar el conocimiento escolar en el marco de su aplicación en el mundo real. En suma, que el profesorado experimente por sí mismo y después recree en el estudiantado la exigencia de pensamiento complejo y autónomo para procesar la información.

Otra finalidad de incluir lectura, escritura, matemáticas y habilidades digitales en la educación escolar tendría que ser aportar elementos de conocimiento y prácticas dirigidas a moldear la personalidad para la convivencia en sociedad desde niveles iniciales hasta en los niveles llamados técnicos o superiores, donde se busca dotar al individuo de elementos 
suficientes de conocimiento y cultura profesional para desempeñarse en determinados escenarios laborales y sociales.

Una parte clave de este proceso será, entonces, incorporar modos de leer, de escribir, de usar los conocimientos matemáticos y los dispositivos digitales de tal manera que se integren como parte del proceso de adquisición y desarrollo de otros contenidos establecidos en la formación escolar. Para propiciar esta alfabetización, en concreto, se propone:

- Incorporar, dentro de la enseñanza, la reflexión sobre las necesidades de comprensión de conocimientos y saberes específicos (alfabetización disciplinar).

- Integrar, de manera estratégica, actividades de lectura, escritura, matemáticas y uso de las TIC en todas las asignaturas o cursos que sean parte de la formación escolar, y en el caso profesional acorde a los usos particulares de la carrera.

- Realimentar las actividades del estudiantado a lo largo del proceso de aprendizaje tanto de los contenidos disciplinares, como de la forma en que la lectura, la escritura, los conocimientos matemáticos y las habilidades digitales están siendo adquiridas.

- Afianzar, en el profesorado, la idea de que la lectura, la escritura, los conocimientos matemáticos y las habilidades digitales no se adquieren de una vez y para siempre, sino que se desarrollan a lo largo de la formación académica, a partir de las experiencias escolares propiciadas en el estudiantado.

\section{Referencias}

Albero, Magdalena. (2002). Adolescentes e Internet. Mitos y realidades de la sociedad de la información. ZER-Revista de estudios de comunicación, 7(13), 1-8. Recuperado de https://www.ehu.eus/ojs/index.php/Zer/article/view/6032/5714

Álvarez-Álvarez, Manuela y Boillos-Pereira, María del Mar. (2015). La producción escrita de los estudiantes universitarios de nuevo ingreso. Magis. Revista Internacional de Investigación en Educación, 8(16), 71-90. doi: http://dx.doi.org/10.11144/Javeriana.m816.peeu

Apolo, Diego, Bayés, Marc y Hermánn, Andrés. (2016).Cambios educativos en los procesos de lectura digital: a pedagogía del ciberespacio como estrategia de procesamiento de contenidos en la era de internet. Redes.com: Revista de estudios para el desarrollo social de la comunicación, (12), 223- 239. Recuperado de https://dialnet.unirioja.es/servlet/articulo?codigo $=5456603$

Argüello, Luis Alfonso. (2009). Cortar y pegar: La producción textual de trabajos escolares universitarios. Questión, 1(21). Recuperado de http://sedici.unlp.edu.ar/bitstream/handle/10915/32240/Documento completo.pdf?sequ $\underline{\text { ence }=1}$ 
Argüello, Luis Alfonso. (2012). Migraciones digitales de lectura y escritura en estudiantes universitarios. Revista de Universidad y Sociedad del Conocimiento, 9(1), 5-21. Recuperado de https://www.redalyc.org/pdf/780/78023415002.pdf

Aparici, Roberto. (2002). Mitos de la educación a distancia y de las nuevas tecnologías. RIED. Revista Iberoamericana de Educación a Distancia, 5(1), 9-27. doi: https://doi.org/10.5944/ried.5.1.1128

Backhoff, Eduardo, Sánchez, Andrés, Peón, Margarita y Andrade, Edgar. (2010). Comprensión lectora y habilidades matemáticas de estudiantes de educación básica en México: 20002005. Revista Electrónica de Investigación Educativa, 12(1). Recuperado de http://redie.uabc.mx/vol12no1/contenido-backhoffsanchez.html

Guevara, Carmen, Cárdenas, Karlena, Reyes, Verónica y González, Claudia. (2017). Niveles de ansiedad y comprensión lectora, en estudiantes mexicanos de escuelas secundarias. Revista Electrónica de Psicología Iztacala, 20(3), 10-57. Recuperado de http://www.revistas.unam.mx/index.php/repi/article/view/61754

Burin, Debora, Coccimiglio, Yamila, González, Federico y Bulla, Jhon. (2016). Desarrollos recientes sobre habilidades digitales y comprensión lectora en entornos digitales. Psicología, Conocimiento y Sociedad, 6(1), 191-206. Recuperado de http://www.scielo.edu.uy/pdf/pcs/v6n1/v6n1a09.pdf

Cabra-Torres, Fabiola, y Marciales-Vivas, Gloria. (2009). Mitos, realidades y preguntas de investigación sobre los "nativos digitales": una revisión. Universitas Psychologica, 8(2), 323-338. Recuperado de https://www.redalyc.org/pdf/647/64712165004.pdf

Calderón-Ibáñez, Arlenys, y Quijano-Peñuela, Jorge. (2010). Características de comprensión lectora en estudiantes universitarios. Revista Estudios Socio-Jurídicos, 12(1), 337-364. Recuperado de https://www.redalyc.org/pdf/733/73313677015.pdf

Carlino, Paula. (2017). Leer y escribir en las ciencias sociales en universidades argentinas. Enunciación, 22(1), 110-124. Recuperado de https://www.aacademica.org/paula.carlino/236.pdf

Flores-Carrasco, Paula, Díaz-Mújica, Alejandro, y Lagos-Herrera, Irma. (2017). Comprensión de textos en soporte digital e impreso y autorregulación del aprendizaje en grupos universitarios de estudiantes de educación. Revista Electrónica Educare, 21(1), 1-17. doi: http://dx.doi.org/10.15359/ree.21-1.7

Castro, Alberto. (2015). Competencias administrativas y académicas en el profesorado de educación media superior. Revista mexicana de investigación educativa, 20(64), 263294. Recuperado de http://www.scielo.org.mx/scielo.php?pid=S1405$\underline{66662015000100013 \& \text { script=sci abstract\&tlng=es }}$

Coiro, Julie. (2011). Predicting reading comprehension on the internet: Contributions of offline reading skills, online reading skills, and prior knowledge. Journal of Literacy Research, 43(4) 352-392. doi: https://doi.org/10.1177/1086296X11421979 
Cuschnir, Paula. (2016). Influencia de la comprensión de textos en la resolución de problemas matemáticos. Exlibris, (5), 495-504. Recuperado de http://revistas.filo.uba.ar/index.php/exlibris/article/download/3041/987

Distéfano, María, Pochulu, Marcel y Font, Vicenç. (2015). Análisis de la complejidad cognitiva en la lectura y escritura de expresiones simbólicas matemáticas. REDIMAT Journal of Research in Mathematics Education, 4(3), 202-233. doi: http://dx.doi.org/10.17583/redimat.2015.1568

Elías, Juan Andrés. (2018). Crónica de una incursión etnográfica a la práctica docente en el bachillerato. Sinéctica: Revista Electrónica de Educación, (51), 1-14. Recuperado de https://www.redalyc.org/pdf/998/Resumenes/Resumen 99859321004 1.pdf

Fernández, Graciela y Carlino, Paula. (2010). ¿En qué se diferencian las prácticas de lectura y escritura de la universidad y las de la escuela secundaria?. LECTURA Y VIDA, 31(3), 6-19. Recuperado de https://www.aacademica.org/paula.carlino/216.pdf

García, Vanessa. (2019). La ambivalencia de la escritura académica entre los profesores de diseño. Foro de Educación, 17(26), 197-218. doi: http://dx.doi.org/10.14516/fde.603

García-Madruga, Juan Antonio y Fernández-Corte, Teresa. (2008). Memoria operativa, comprensión lectora y razonamiento en educación secundaria. Anuario de Psicología, 39(1), 133-157. Recuperado https://www.raco.cat/index.php/anuariopsicologia/article/view/99799

Godino, Juan, Font, Vicenç, y Wilhelmi, Miguel. (2008). Análisis didáctico de procesos de estudio matemático basado en el enfoque ontosemiótico. Publicaciones , 38, 25-48. Recuperado de http://revistaseug.ugr.es/index.php/publicaciones/article/view/2245

Godoy, Lucía. (2017). Fotografías en el aula: ¿nuevas prácticas de lectura y escritura mediadas por TIC?. QUADERNS DIGITAL.NET, 85, 108-125. Recuperado de https://ri.conicet.gov.ar/handle/11336/75518

González, Yolanda, Jiménez, José, y Rosas, Jorge. (2016). Prácticas lectoras de estudiantes universitarios con fines de escritura académica. Revista Electrónica Actualidades Investigativas en Educación, 16(1), 1-19. doi: http://dx.doi.org/10.15517/aie.v16i1.21971

Grasso, Mauricio, Pagola, Lila, y Zanotti, Agustín. (2017). Políticas de inclusión digital en Argentina. Usos y apropiaciones dentro y fuera de la escuela. Pixel-Bit. Revista de Medios y Educación, (50), 95-107. Recuperado de https://www.redalyc.org/pdf/368/36849882006.pdf

Ibarra, Luz, Escalante, Ana, y Darío, César. (2014). Obstáculos para la formación docente en la educación media superior en México. El caso del bachillerato tecnológico. Revista Electrónica Diálogos Educativos, 14(27), 21-54. Recuperado de http://dialogoseducativos.umce.cl/revistas/n27/ibarra.pdf 
Jiménez, José. (2011). Conceptos matemáticos en estudiantes de bachillerato. ¿Qué queda al final del desarrollo de un plan de estudio? Revista de Educación y Desarrollo, (18), 59-66. http://www.cucs.udg.mx/revistas/edu desarrollo/anteriores/18/018 Jimenez.pdf

Lugo de Usategui, Kenia. (2005). El proceso de lectura de hipertextos, ¿una nueva forma de leer? EDUCERE, 9(30), 365-372. Recuperado de https://www.redalyc.org/pdf/356/35603012.pdf

Machaba, France. (2018). Pedagogical demands in Mathematics and Mathematical Literacy: A case of Mathematics and Mathematical Literacy teachers and facilitators, EURASIA Journal of Mathematics, Science and Technology Education, 14(1), 95-108. doi: https://doi.org/10.12973/ejmste/78243

Moreno, Emilce. (2019). Lectura académica en la formación universitaria: tendencias en investigación. Lenguaje, 47(1), 91-119. doi: https://doi.org/10.25100/lenguaje.v47i1.7180

Organización para la Cooperación y el Desarrollo Económico (OECD por sus siglas en inglés). (2919). Resultados de PISA 2018, Volumen I-III. Paris: PISA, OECD Publicaciones. Recuperado de https://www.oecd-ilibrary.org/education/pisa-2018-results-volumei 5f07c754-en;jsessionid=2QqoOv1eF2BcZ7NiXONUG3 F.ip-10-240-5-137

Peredo, María. (2016). Lectura y ciencia en diversos posgrados y disciplinas. Revista de la educación superior, 45(180), 41-54. Recuperado de http://dx.doi.org/10.1016/j.resu.2016.07.001

Rocha, María Alejandra. (2004). El lenguaje de los jóvenes en el chat. Estudios sobre las culturas contemporáneas, 10(19),109-140. Recuperado de https://www.redalyc.org/pdf/316/31601907.pdf

Sandoval, Pedro, Frit, Miguel, Maldonado, Ana, y Rodríguez, Francisco. (2010). Evaluación de habilidades en matemática y comprensión lectora en estudiantes que ingresan a pedagogía en educación básica: un estudio comparativo en dos universidades del Consejo de Rectores. Educar em Revista, (2), 73-102. Recuperado de https://www.redalyc.org/pdf/1550/155021686005.pdf

Secretaría de Educación Pública (SEP). (2017). Modelo educativo para la educación obligatoria ( $2^{a}$ ed.). Ciudad de México, México: Secretaría de Educación Pública. Recuperado https://www.gob.mx/cms/uploads/attachment/file/207252/Modelo Educativo OK.pdf

Serrano, María, Duque, Yolimar, y Madrid, Alix. (2012). Prácticas de escritura académica en la universidad: ¿reproducir o transformar? Educere, 16(53), 93-108. Recuperado de https://www.redalyc.org/pdf/356/35623538011.pdf

Triana, Luz. (2015). Diagnóstico de las competencias lectoescritas en estudiantes de posgrado. Academia y Virtualidad, 8(1), 76-87. Recuperado de https://revistas.unimilitar.edu.co/index.php/ravi/article/view/449/243 
Ugarriza, Nelly. (2006). La comprensión lectora inferencial de textos especializados y el rendimiento académico de los estudiantes universitarios del primer ciclo. Persona, (9), 31-75. Recuperado de https://www.redalyc.org/pdf/1471/147112814002.pdf

Uribe-Álvarez, Graciela y Camargo-Martínez, Zahyra. (2011). Prácticas de lectura y escritura académicas en la universidad colombiana. Magis. Revista Internacional de Investigación en Educación, 3(6), 317-341. Recuperado de https://www.redalyc.org/pdf/2810/281021734005.pdf 
Revista indizada en

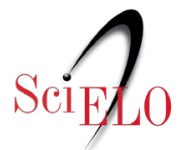

redalyc.sy latindex

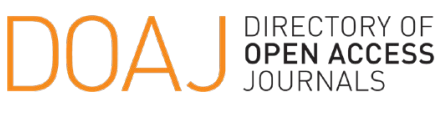

Distribuida en las bases de datos:
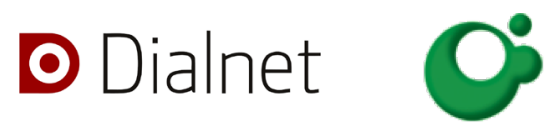
SHERPA/RøMEO

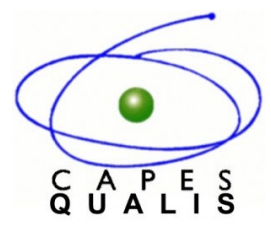

MIAR 